\title{
Keratan Sulfate, a "Unique" Sulfo-Sugar: Structures, Functions, and Synthesis
}

\author{
Masashi Ohmae; Yuji Yamazaki; Kyohei Sezukuri; and Junko Takada \\ Department of Material Chemistry, Graduate School of Engineering, Kyoto University, Nishikyo-ku, Kyoto 615-8510, Japan \\ FAX: +81-75-383-2401, E-mail: ohmae.masashi.4n@kyoto-u.ac.jp
}

(Received on April 23, 2019, accepted on May 16, 2019)

Key Words: sulfo-sugars, keratan sulfate, keratanase, transglycosylation, sugar oxazolines

\begin{abstract}
Keratan sulfate is a sulfated polysaccharide classified as a glycosaminoglycan, which has the structurally unique characteristics of diversity in the linker oligosaccharides connecting to the core protein, the existence of both an intrachain fucose branch and a "capping" monosaccharide at the nonreducing end, and diversity of the sulfation patterns. The function(s) of this newest glycosaminoglycan, keratan sulfate, remain mostly unclear. In this minireview, we describe the structures and known functions of keratan sulfate as well as cutting-edge methods to enable the synthesis of keratan sulfate with complex structures.
\end{abstract}

\section{A. Introduction}

Sulfated sugars are widely distributed in many living systems, from plants to animals, and exist in various forms from low-molecular-weight glycosides to polysaccharides (1-4). Many of these sulfo-sugars exhibit their biological functions through interactions with specific proteins; a sulfate group often functions as the key for these interactions. In the animal kingdom, almost all sulfo-sugars are found as glycosaminoglycans (GAGs), which include polysaccharide members consisting of hyaluronan (HA), heparan sulfate (HS)/heparin (Hep), chondroitin sulfate (CS)/dermatan sulfate (DS) and keratan sulfate (KS) (Fig. 1) (3). GAGs are linear copolymers of alternating uronic acids (glucuronic acid and iduronic acid) and hexosamines [glucosamine $(\mathrm{GlcN})$ and galactosamine], which have sulfate groups $(\mathrm{O}$ - and/or $\mathrm{N}$-) at varying frequencies (except HA); however, KS contains Gal instead of a uronic acid, and often an $\alpha$-Fuc branch at the $\mathrm{C} 3$ of GlcNAc. GAGs except HA exist as

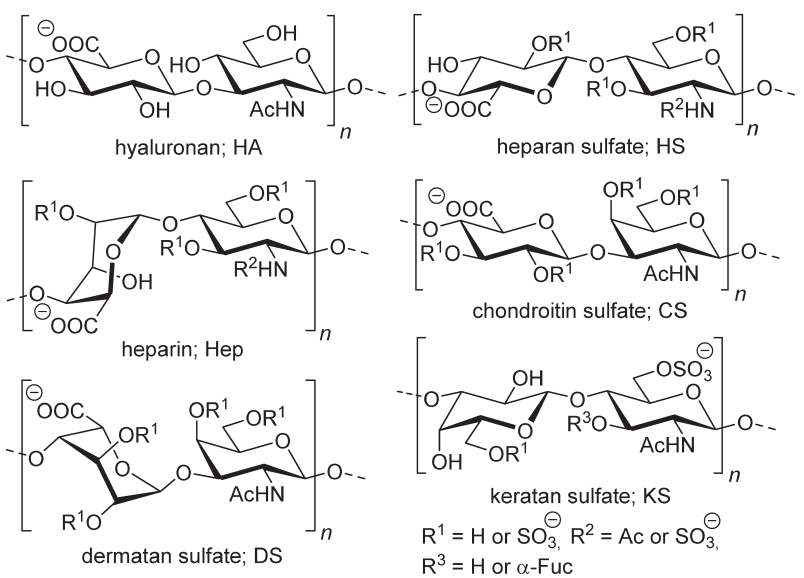

Fig. 1. Typical structures of the repeating disaccharides found in GAGs as the representative sulfo-sugars. the carbohydrate parts of glycoproteins called proteoglycans (PGs) that connect to core proteins through "linker" oligosaccharides (HS/Hep and CS/DS: $O$-glycans, KS: $N$ - and $O$-glycans). In Fig. 1 , disaccharide structures are shown as the typical repeating units of GAGs, but these have additional sulfation patterns. In addition, CS contains DS units at varying ratios, while HS also contains Hep units, and vice versa. Thus, taking these sulfation patterns into account, it becomes clear that GAGs have extremely complex, heterologous structures even within a GAG class; this makes investigating their functions extremely difficult. Despite these conditions, significant knowledge has been revealed through the efforts of many researchers, especially in the cases of HA (5), HS (6) and CS (7). In contrast, few research reports have been published on KS as compared to other GAGs since its discovery in 1939 (8), and it has mostly into oblivion. In this mini-review, the state-of-the-art results from these "old and new" KS studies, along with future perspectives, are discussed.

\section{B. Keratan Sulfate}

\section{B-1. Structures and Classification}

As described above, KS contains Gal residues instead of uronic acids; a unique difference from other GAGs. Because of this, KS has a type II polylactosamine chain consisting of $\mathrm{Gal}$ and GlcNAc alternately connecting through $\beta(1 \rightarrow 4)$ - and $\beta(1 \rightarrow 3)$ glycosidic linkages, respectively; the C6 of GlcNAc is always sulfated (9). In addition to this basic structure, sulfation of the C6 of Gal, $\alpha$-L-fucosylation of the $\mathrm{C} 3$ of GlcNAc, and the addition of the "capping" monosaccharide $(\alpha-\mathrm{Neu} 5 \mathrm{Ac}, \alpha$-Neu5Gc, $\alpha$-Gal, $\beta$-GlcNAc6S, $\beta$-GalNAc6S) at the non-reducing end are all factors which provide structural diversity to KS (Fig. 2). KS exists as a side chain of PGs, and is divided into three types: KS-I, KS-II, and 


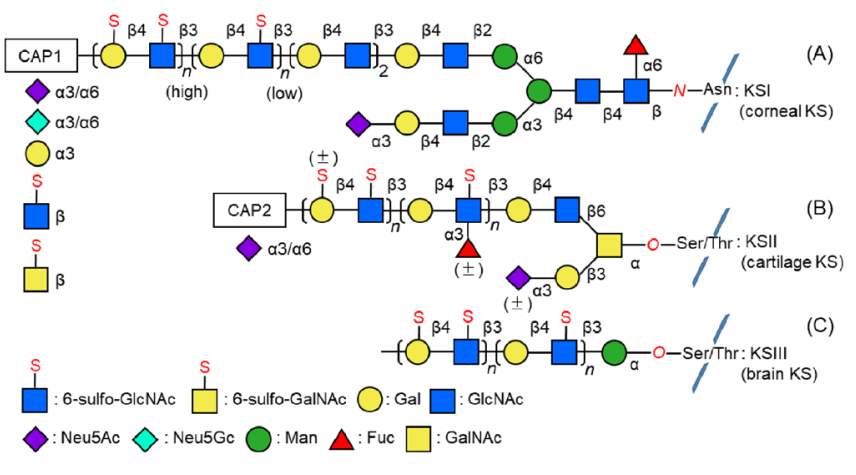

Fig. 2. The typical structures of three KS types. (A) KS-I (corneal KS), (B) KS-II (cartilage KS), (C) KS-III (brain KS).

KS-III, based on the structural differences of the linker oligosaccharides connecting the protein core to KS. These KS types are frequently found in the cornea, cartilage, and brain, respectively; therefore, they are alternatively called corneal KS, cartilage KS and brain $\mathrm{KS}$.

KS-I binds to an Asn side chain on the core proteins through its complex-type $N$-glycan, and is normally found on the branching chain of the C2 of $\alpha 6$-Man. KS-I has higher amounts of a disulfated LacNAc unit containing 6-sulfo-Gal (Fig. 1, $\mathrm{R}^{1}=\mathrm{SO}_{3}^{-}$in $\mathrm{KS}$ ), to which the aforementioned capping monosaccharide is frequently added, at the non-reducing end.

The linker oligosaccharide of KS-II is a core II type $O$-glycan, which connects to a Ser or Thr side chain on the core protein. The $\beta 6$-GlcNAc branch on the core II oligosaccharide contains the KS chain; however, KS-II is shorter than KS-I, and the C3 of the 6-sulfo-GlcNAc residue is often substituted with $\alpha$-Fuc, leading to the formation of a 6-sulfo-Lewis X (su-LeX) structure. The nonreducing terminal is capped by sialylation.

The newest type of KS is KS-III, which connects to a Ser or Thr side chain on the core protein using $\alpha$-mannoside as the linker. Its detailed structure, however, has not yet been elucidated.

\section{B-2. Biosynthesis}

There are several points in the process of KS biosynthesis that should be discussed. It is believed that the linker oligosaccharides are constructed according to the well-known biosynthesis systems of $N$ - $(10,11)$ and $O$-glycans. The transferases involved in the synthesis of the KS main chain, the 6-sulfo-LacNAc polymer, are well studied (Fig. 3). In the biosynthetic path of KS, $\beta(1 \rightarrow 3)$ $N$-acetylglucosaminyltransferase $(\beta 3 \mathrm{GnT})(12)$ adds a GlcNAc residue to the non-reducing terminal $\mathrm{Gal}$ in the growing chain of both KS-I and KS-II. Then, the specific sulfotransferase catalyzes the addition of a sulfate group at the $\mathrm{C} 6$ of the non-reducing terminal GlcNAc. Five kinds of the GlcNAc-6-O-sulfotransferases (GlcNAc6STs) have been cloned in humans to date (13); GlcNAc$6 \mathrm{ST}-5,-3$, and -1 are the sulfotransferases responsible for KS sulfa-

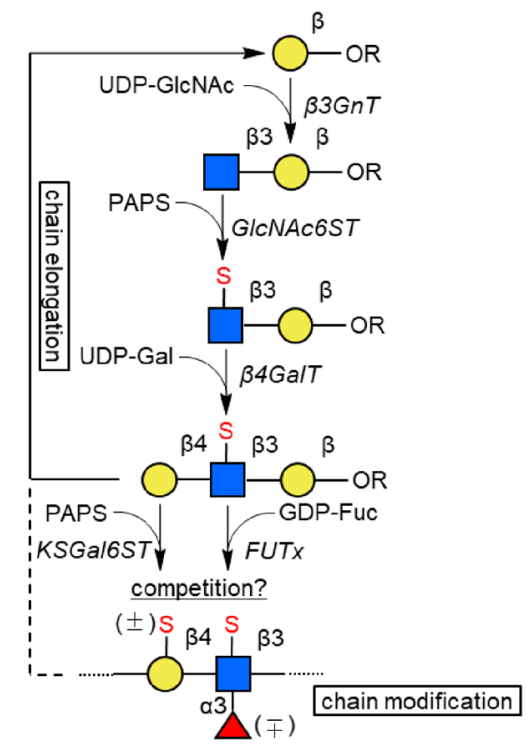

Fig. 3. Biosynthetic path of KS and related transferases.

tion in the cornea $(14,15)$, cartilage $(16)$, and brain (17), respectively, though their substrate specificities often overlap (16). To the resulting 6-sulfo-GlcNAc at the non-reducing terminal, a Gal residue is then added through the $\beta(1 \rightarrow 4)$-glycoside. Among the $\beta(1 \rightarrow 4)$-galactosyl transferases ( $\beta 4 \mathrm{GalTs}), \beta 4 \mathrm{Gal}-\mathrm{T} 1$ is involved in the formation of the non-sulfated LacNAc polymer (18). Intriguingly, $\beta 4 \mathrm{Gal}-\mathrm{T} 4$ prefers to transfer a Gal residue when the GlcNAc at the non-reducing end contains a sulfate group at C6 (19); therefore, this enzyme is believed to be the primary $\beta 4 \mathrm{GalT}$ responsible for KS biosynthesis. Thus, these three enzymes: $\beta 3 \mathrm{GnT}$, GlcNAc$6 \mathrm{ST}$, and $\beta 4 \mathrm{Gal}-\mathrm{T}$, cooperate to construct the basic structure of KS. Sulfation of the $\mathrm{C} 6$ of Gal, which is one of the factors providing structural diversity to $\mathrm{KS}$, is carried out by the catalysis of keratan sulfate galactose 6-sulfotransferase (KSGal6ST) (20) or chondroitin sulfotransferase-1 (C6ST-1) (21), both of which have low transferase activity. Particularly, the activity of KSGal6ST decreases drastically when the neighboring GlcNAc has no sulfate group (22). Another factor involved in the structural diversity of $\mathrm{KS}$ is the addition of $\alpha$-Fuc at the C3 of GlcNAc. Six kinds of $\alpha(1 \rightarrow 3)$ fucosyltransferases (FUTs) have been identified in humans; however, the FUT responsible for $\alpha$-fucosylation at the internal GlcNAc in KS has not yet been determined $(23,24)$. KSGal6ST seems to have difficulty transferring a sulfate group at the C6 of Gal when the neighboring GlcNAc has a $\alpha(1 \rightarrow 3)$-Fuc residue, and the 6,6'-di-sulfo-LeX structure is difficult to find in KS; therefore, the FUT(s) involved in KS biosynthesis might struggle to transfer $\alpha$-Fuc to the C3 of GlcNAc when the subsequent Gal has a sulfate group at C6 (24). Furthermore, there are no reports that describe transferases catalyzing the addition of capping monosaccharides at 
the non-reducing end of KS.

\section{B-3. Biological Activities}

$\mathrm{KS}$ is widely distributed, as keratan sulfate proteoglycans (KSPGs), in the endothelia, the central and the peripheral nervous systems (CNS, PNS), in addition to the cornea, cartilage, and brain, where its functions are well studied. As described above, three types of KS show further structural diversity derived from sulfation and $\alpha$-fucosylation, resulting in the formation of extremely complex structures. This complexity presents a challenge for revealing the relationship between KS microstructures and its functions. Aggrecan (25), fibromodulin (26) and keratocan (26) found in connective tissues like cartilage, MUC1 (27) in mucin, Abakan (28), synapse vesicle protein 2 (SV2) (29) and phosphacan (30) in CNS/PNS are all known KSPGs. KS biosynthesis in the cornea occurs during the early embryonic stage, where the degree of sulfation increases with cellular maturation. The KSPGs with highly sulfated KS (decorin, lumican, mimecan, and keratocan) critically regulate the size of collagen fibrils and appropriate interfibrillar spacing, leading to the formation of corneal tissue with remarkable transparency and optical clarity (31). The overlapping functions of KS and CS in the CNS have been reported (32); the KS chain inhibits neural plasticity by impeding axon regeneration. Recently, fucosylated KS with sialic acid capping (Fig. 4A) has been found in the microglia and seems to be a factor for malignancy in the neurodegenerative diseases such as amyotrophic lateral sclerosis (ALS) and Alzheimer's disease $(33,34)$. Furthermore, $\mathrm{KS}$ on aggrecan extracted from human tracheal submucosal glands has $\alpha 2,3$-sialylated chains, which are predicted to work as ligands
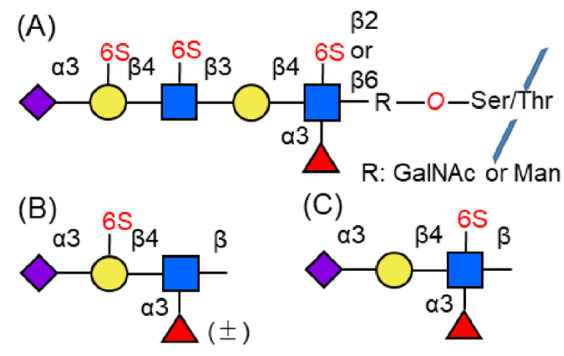

(C)
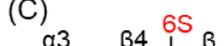

(D)

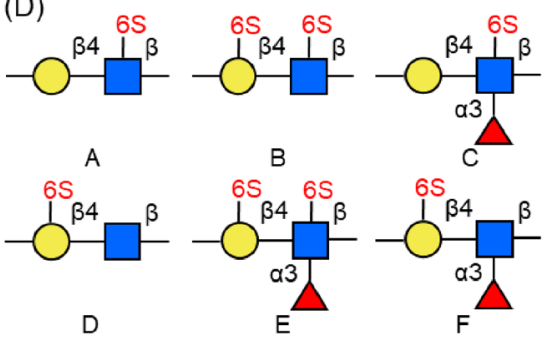

Fig. 4. Structures of disease-related KS oligosaccharides. (A) ALS and Alzheimer's disease, (B) the ligand for Siglec-8, (C) the ligand for "lymphocyte homing" and Siglec-9, and (D) definition of the structurally possible units (A-F units) of KS. to Siglec-8 on eosinophils that contribute to asthma (35); the interaction of Siglec- 8 with its ligands can induce apoptosis of the leukocyte. Glycan array screening of Siglec- 8 clearly shows that the best ligand candidates are sialyl 6'-sulfo-LeX (Fig. 4B) and sialyl $6^{\prime}$-sulfo-LacNAc; however, there is no data that the sulfate group exists exclusively at the $\mathrm{C} 6$ of $\mathrm{Gal}$ in $\mathrm{KS}$. Taken together, these data suggest that 6,6'-disulfated KS counterparts are the "real" ligands. In addition, sialyl 6-sulfo-LeX (Fig. 4C), which is a well-known ligand found in high endothelial venules for L-selectin signaling during "lymphocyte homing" (36), may be a ligand for Siglec-9 expressed on neutrophils and tumor-infiltrating $\mathrm{CD}^{+} \mathrm{T}$ cells in cancer tissues like non-small cell lung cancer; decreases $\mathrm{CD}^{+} \mathrm{T}$ cell activity, resulting in immune evasion by tumor cells (37).

The functions mentioned above behave similarly to known immune checkpoints; therefore, sialylated KS oligosaccharides have potential as new targets for drug design against autoimmune diseases. Thus, sialylated KS-related oligosaccharides are critical molecules that regulate vital events, particularly in the immune system (immune suppression), through interactions with Siglecs. In addition, KS is expressed on ES and iPS cells, and may be an index for distinguishing whether the cell is a carcinoma cell by using specific antibodies (38).

\section{Synthesis of KS Oligosaccharides}

KS synthesis with regulated sulfation patterns is essential for the utilization of highly functional KS chains because of the importance of sulfation status for interaction with Siglecs and lectins, although as mentioned above a detailed relationship between KS microstructures and their functions has not been described. The importance of the regulation of sulfation patterns has come to be known as the "sulfation codes" for GAGs, as reported in a study on CS and HS functions (39). As $\alpha$-fucosylation is the other factor providing structural diversity to KS, a synthesis method that can control both of these factors (patterns of sulfation and $\alpha$-fucosylation) must be developed, as well as including modification by the capping sugars, typically sialic acid, at the nonreducing terminal. The structural possibilities for $\mathrm{KS}$ are defined hereafter as units A through F, for simplicity of explanation (Fig. 4D).

\section{C-1. Organic Synthesis}

Organic synthesis is the first choice for carbohydrate synthesis. In KS synthesis, tetrasaccharide (1) was successfully synthesized by this method $(40,41)$ (Fig. 5A).

All of the glycosidic linkages in the KS main chain are 1,2-trans-glycosides; therefore, in the molecular design of GlcN and Gal derivatives at the reducing end of the glycosyl donors, 

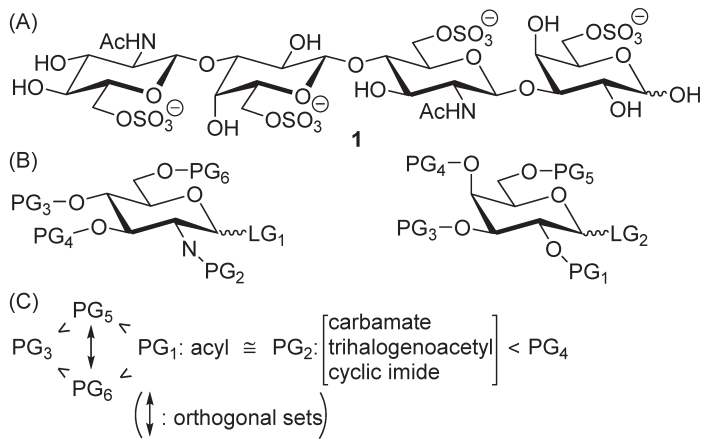

Fig. 5. (A) The KS tetrasaccharide prepared by organic synthesis for the first time, (B) protecting groups for effective organic synthesis of $\mathrm{KS}$ and $(\mathrm{C})$ their reactivity order.

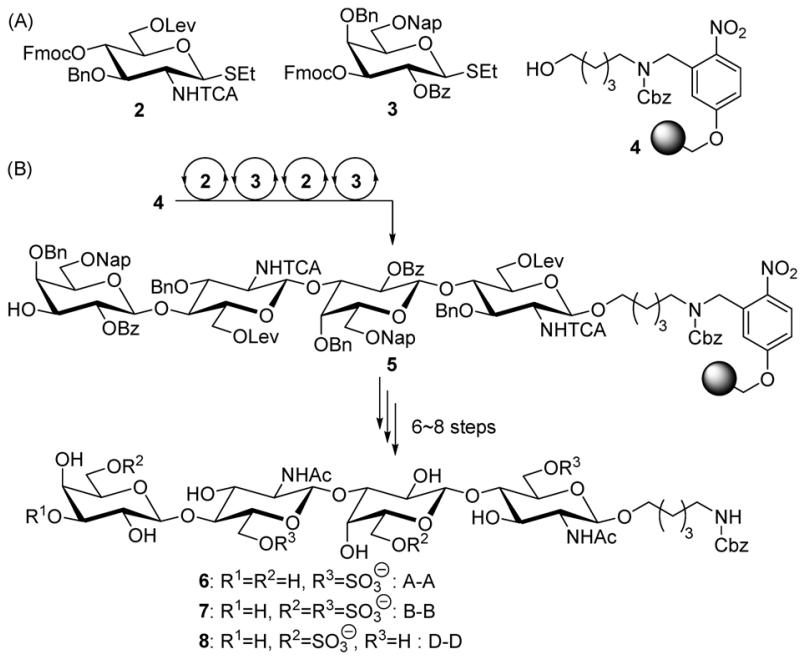

Fig. 6. (A) The glycosyl donors with rationally designed sets of protecting groups (2 and 3 ) and the solid support (4) for solidphase synthesis, (B) the solid-phase synthesis of tetrasaccharides (6-8) with uniform sulfation patterns using 2-4. [Reorganized from Hahm, H. S., Broecker, F., Kawasaki, F., Mietzsch, M., Heilbronn, R., Fukuda, M., Seeberger, P. H. (2017) Chem 2, 114-124. Copyright $\odot$ 2017, with permission from Elsevier].

the protecting groups at $\mathrm{C} 2$ must not only work as effectively as the neighboring participating groups, but also be removed under mild conditions. Acyl protections fit these conditions, particularly in GlcN derivatives $\left(\mathrm{PG}_{2}\right)$, carbamates (Troc, $\mathrm{Cbz}$ ), trihalogenoacetyls (TFA, TCA), and cyclic imides (Phth, TCP) which are frequently employed as $\mathrm{PG}_{2}$ (Fig. 5B). On the hydroxy groups, other than $\mathrm{C} 1$ bearing a leaving group, the following protecting groups must be designed as a set to provide ordered removability: the $\mathrm{C} 4$ in GlcN and the $\mathrm{C} 3$ in Gal, the two C6s of both GlcN and $\mathrm{Gal}$ for sulfation, and the remaining $\mathrm{C} 3$ and $\mathrm{C} 4$ of GlcN and Gal, respectively (Fig. $5 \mathrm{C}$ ). The leaving groups $\left(\mathrm{LG}_{1}, \mathrm{LG}_{2}\right)$ that activate anomeric carbons must be stable under the $\mathrm{PG}_{3}$ removal conditions. There may be other designs that follow the above limitations, but a successful study on the solid-phase synthesis of KS oligosaccharides by using GlcN (2) and the Gal (3) derivatives designed through rational protecting group selection has recently been reported (Fig. 6) (42). The protecting groups in $\mathbf{2}$ and $\mathbf{3}$ are all in accordance with the reactivity order shown in Fig. 5C, and $\mathrm{KS}$ tetrasaccharides A-A (6), B-B (7), and D-D (8), all of which have uniform sulfation patterns, have been successfully synthesized by this method.

\section{C-2. Synthesis by Transferases}

There has been some success identifying the transferases responsible for biosynthesis of the KS main chain, and KS synthesis using these transferases has been achieved $(15,43)$. Particularly, KS oligosaccharides were made in 2007 when the enzyme mixture of $\beta 3 \mathrm{GnT} 7, \beta 4 \mathrm{Gal}-\mathrm{T} 4$ and GlcNAc6ST-5 was reacted with a mixture of the ${ }^{35} \mathrm{~S}$-labeled acceptor trisaccharide [GlcNAc6 ${ }^{35} \mathrm{~S} \beta(1 \rightarrow 6)$ $\operatorname{Man} \alpha(1 \rightarrow 6)$ Man $\alpha$-octyl], UDP-GlcNAc, UDP-Gal and PAPS, which gave rise to KS oligosaccharides, from disaccharides to octasaccharides, possessing various sulfo-forms at the C6 of GlcNAc following the trisaccharide acceptor (Fig. SI-1). The synthesis of KS oligosaccharides with a uniform structure, however, has not yet been achieved.

\section{C-3. Chemo-Enzymatic Synthesis}

As described above, linear KS oligomers have been exclusively prepared by organic synthesis or transferase-catalyzed biosynthesis. KS, however, is structurally diverse not only in its sulfation patterns, but also in $\alpha$-fucosylation at the $\mathrm{C} 3$ of GlcNAc, and in non-reducing terminal capping (9). Therefore, novel methods to synthesize KS with these structural diversities must be developed to fully reveal the functions of naturally occurring KS. Due to a decrease in reactivity of the glycosyl donor and acceptor as chain length increases, KS synthesis by organic chemistry becomes difficult. Sulfotransferases, FUTs and the transferases responsible for adding the capping sugars remain unidentified to date, which makes the biochemical synthesis of KS difficult. In these situations, chemo-enzymatic synthesis, which integrates the advantages of both organic and biochemical methods, exhibits superior performance. In the following section, the chemo-enzymatic synthesis of KS oligomers that allows for the possibility of structural diversity is introduced.

To date, we have successfully achieved the synthesis of GAGs by utilizing the transglycosylation activities of glycoside hydrolases (44); particularly in KS synthesis, keratanase II (KSase II), which is one of the endo-type KS hydrolases, has been employed (45-47). In order to induce transglycosylation reactions effectively, activated glycosyl substrates must be used as glycosyl donors (48); typically, glycosyl fluorides or glycosyl oxazolines have been adopted depending on the catalysis mechanism of the enzymes.

$\mathrm{KSase}$ II is one of the endo- $\beta$ - $N$-acetylglucosaminidases 
responsible for the hydrolysis of the $(1 \rightarrow 3)-\beta-6-O$-sulfo- $N$ acetylglucosaminide linkage (49). Although the catalysis mechanism of this enzyme has not yet been identified, we predict that the enzyme has a "substrate-assisted mechanism" containing an oxazolinium ion intermediate during catalysis, which many retainingtype $\mathrm{N}$-acetylhexosaminidases (50) possess. According to this prediction, we first prepared glycosyl oxazoline derivatives (9 and 10) with the basic units $A$ and $B$ from KS, respectively (Scheme 1) (45). These donors were subjected to reactions with KSase II isolated from Bacillus sp. Ks36 (abbreviated as Ks36), which produced the oligomers of A (12) from 9, and the tetrasaccharide corresponding to the $\mathrm{B}$ dimer (13) from $\mathbf{1 0 .}$

These results strongly suggest that Ks36 is a processive enzyme (51) with a "substrate-assisted mechanism" through an oxazolinium ion intermediate, which can effectively catalyze transglycosylation. The substrate specificities of Ks36 in hydrolysis reactions have been extensively studied $(52,53)$. Based on the results of these studies, we further anticipated that the enzyme could recognize a $\mathrm{C}$ unit (su-LeX) with an $\alpha$-Fuc residue at $\mathrm{C} 3$ of GlcNAc6S in the subsites (54) next to the catalytic center. In order to confirm the enzyme ability, we carried out a reaction of the newly designed su-LeX oxazoline derivative (11) with Ks36. The oxazoline donor of $\mathbf{1 1}$ was effectively recognized by Ks36, and produced the corresponding C unit oligomers (14A) (46). It should be pointed out that KSase II from Bacillus circulans KsT202 (abbreviated as KsT202) has also been isolated (55), and can catalyze the transglycosylation of $\mathbf{1 1}$ to the corresponding $\mathrm{C}$ unit dimer (14B) as a sole product (46).

The substrate specificities of KsT202 have, however, not been sufficiently studied. Therefore, we investigated this using transglycosylation reactions (47). As shown in Scheme 1, KsT202 produced the $\mathrm{C}$ unit dimer as the sole transglycosylation product by using 11; this result urged us to investigate whether KsT202 has similar processivity to Ks36 by carrying out the reaction of the least structurally hindered oxazoline derivative of the A unit (9) with KsT202. The reaction of 9 with KsT202 proceeded smoothly, and provided the corresponding oligomers 12 containing dodecasaccharides $(n=6)$, or larger in high yields. In contrast, the transglycosylation of the $6,6^{\prime}$-disulfo-LacNAc oxazoline derivative (10) produced the corresponding tetrasaccharide $(13 ; n=2)$ as the sole product. These results are in complete agreement with those from Ks36; therefore, KsT202 has similar specificity for substrates and reactions. In addition, two of the reactions, namely $\mathbf{1 0}$ with the $\mathrm{C}$ unit (Fig. SI-2A), and $\mathbf{1 1}$ with the A unit, produced the A-C and the $\mathrm{C}-\mathrm{A}$ oligosaccharides as the sole products of single glycosylation, respectively. These results allowed us to make a new prediction about substrate recognition around the subsite $(+4)$ and the cata-

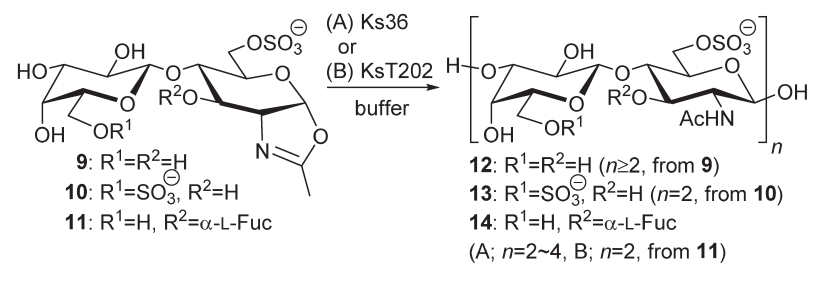

Scheme 1. Transglycosylation of the sugar-oxazoline derivatives catalyzed by KSase II.

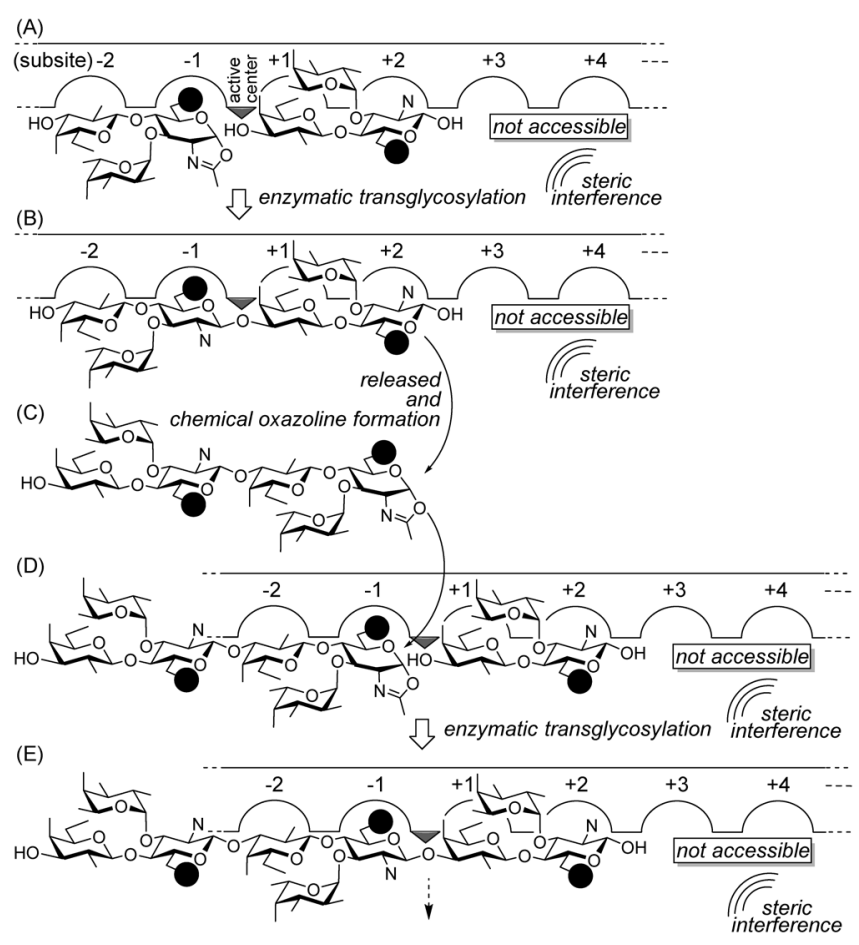

Fig. 7. A new concept for KS chain elongation. (A-B) a single transglycosylation of an oxazoline donor and a free acceptor, (B-C) chemical oxazoline formation of a glycosylation product, (C-D-E) a single transglycosylation of a newly prepared oxazoline donor and a free acceptor. The hydroxy groups of C2, C4 and C6 of Gal, C3 of GlcNAc, and C2-C4 of Fuc are omitted. C2 acetamide and sulfate are symbolized as $\mathrm{N}$ and filled circles, respectively.

lytic center, which have structures interfering with the processive sliding of the substrate sugars. Thus, due to steric interference by the sulfate group at $\mathrm{C} 6$ of Gal, or the $\alpha$-Fuc residue at the $\mathrm{C} 3$ of GlcNAc6S against the enzyme, we predicted that transglycosylation of either the $\mathrm{B}$ unit oxazoline derivative (10) or the $\mathrm{C}$ unit oxazoline derivative (11) had produced the corresponding dimer $(n=2)$ as the main product in each case (Fig. SI-2B-C).

The results obtained above strongly imply that synthesis of KS oligomers with an internal Gal6S and/or $\alpha$-Fuc is difficult to obtain using a methodology where chain elongation is carried out by the addition of the sugar unit to the non-reducing end, similar to what we have used historically. Thus, we have switched to the idea that KS chain elongation be performed at the reducing end, in a 


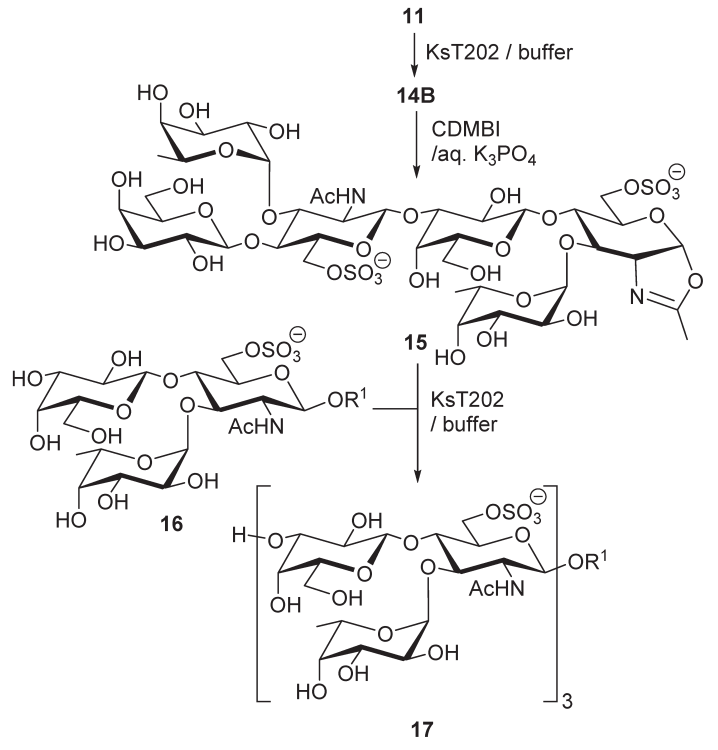

Scheme 2. Synthesis of the su-LeX trimer derivative on the basis of the new concept. $\mathrm{R}^{1}:\left(\mathrm{CH}_{2} \mathrm{CH}_{2} \mathrm{O}\right)_{3} \mathrm{CH}_{2} \mathrm{CH}_{2} \mathrm{~N}_{3}$.

direction opposite to the previous method utilizing the enzyme processivity (Fig. 7); namely, KsT202-catalyzed glycosylation of the oxazoline donor derivative with a free $\mathrm{KS}$ unit acceptor is carried out $(\mathrm{A} \rightarrow \mathrm{B})$, and the produced oligosaccharide is then chemically converted to the corresponding oxazoline derivative $(B \rightarrow C)$. The newly prepared oxazoline derivative is glycosylated with a free KS unit by KsT202 $(\mathrm{C} \rightarrow \mathrm{D} \rightarrow \mathrm{E})$. Thus, step-by-step chain elongation synthesis of KS oligosaccharides along with control of the unit alignment can be achieved using this series of these reactions. In this new methodology, neither subsites over $(+3)$ with limited substrate specificities nor enzymes with a processive nature are needed, whereas the chemical oxazoline conversion of the produced KS oligosaccharide is essential for re-activation. As a method for oxazoline formation of free sugars using DMC (2-chloro-1,3-dimethylimidazolinium chloride) or CDMBI (2-chloro-1,3-dimethyl-1 $H$ benzimidazol-3-ium chloride) as an activator has been established (56), we carried out oxazoline formation using this method.

According to this concept, synthesis of the $\mathrm{C}$ unit trimer, which is difficult to synthesize using our previous method, was first investigated (Scheme 2). The $\mathrm{C}$ unit dimer (14B), which can be easily obtained by transglycosylation of the $\mathrm{C}$ unit oxazoline derivative (11), was converted to the corresponding $\mathrm{C}$ unit dimer oxazoline derivative (15) by using CDMBI. Transglycosylation of $\mathbf{1 5}$ using a su-LeX derivative with an azido-functionalized linker (16) (57) as the glycosyl donor and the glycosyl acceptor, respectively, was carried out with KsT202. As we expected, the reaction proceeded very smoothly, and gave rise to the $\mathrm{C}$ unit trimer derivative (17) with good yields (manuscript in preparation). This result is in sharp contrast with the previous result that the reaction of $\mathbf{1 1}$ could

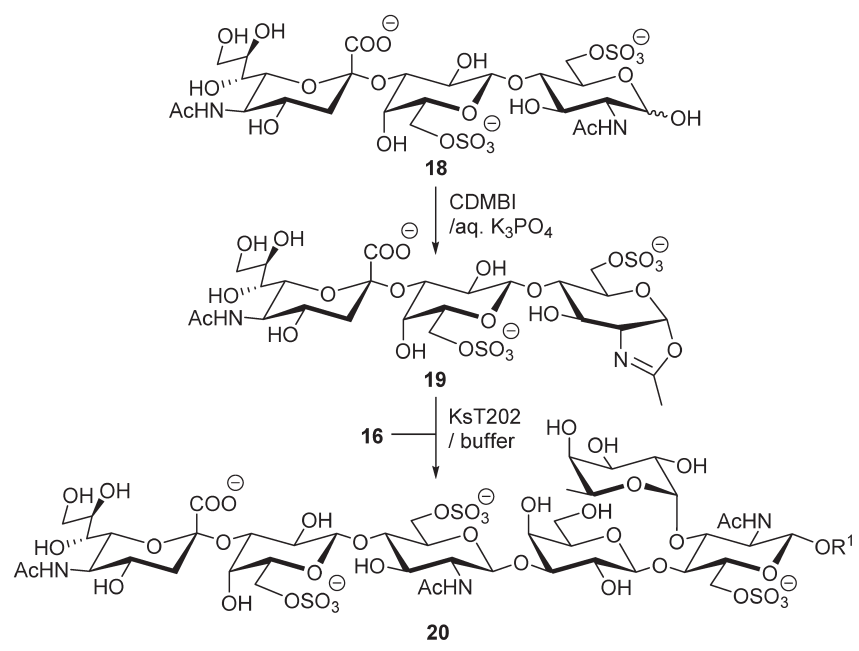

Scheme 3. Synthesis of the KS oligosaccharide with a "capping" sugar on the basis of the new concept.

not provide the $\mathrm{C}$ unit trimer at all. Therefore, this result strongly supports the use of enzymatic reactions as conceptually proposed above.

The above result also suggests that the subsites for the glycosyl donors (the "-" subsites) have fewer limitations for substrate recognition compared to those for the glycosyl acceptors (the "+" subsites). Thus, we have tried to synthesize KS oligosaccharide derivatives with a "capping" sugar (Scheme 3); a sialic acid (Nue5Ac) residue was selected as the "capping" sugar, which is frequently found at the non-reducing ends of the various glycan chains and is involved in interactions with in- and/or ex vivo substances. We first tried to synthesize the KS hexasaccharide shown in Fig. 4A (manuscript in preparation). The free $\alpha(2,3)$-sialyl6,6'-di-sulfo-LacNAc (18) synthesized chemically was treated with CDMBI. The obtained oxazoline derivative 19 was reacted with the su-LeX derivative $\mathbf{1 6}$ by transglycosylation catalyzed by KsT202. As we expected, this reaction produced the target sialoKS oligosaccharide (20).

Thus, on the basis of this concept, a step-by-step KS chain elongation that controls not only the "sulfation code," but also the substitution patterns of $\alpha$-Fuc, selection of the "capping" sugar, and the aglycon structure, has been achieved by appropriate selection of the KS unit. Next, we are planning to expand this concept to the synthesis of longer KS oligosaccharides with other "capping” sugars.

\section{Future Perspectives}

The synthesis of sequence-regulated KS oligosaccharides with "capping" sugars has been successfully achieved by KSase IIcatalyzed chemoenzymatic synthesis. This method will enable us to reveal the detailed structures of the bioactive KS oligosaccharides described in section B-3. Furthermore, the final step of this method 
is an enzymatic reaction, which needs no extra-chemical reactions (such as removal of protecting groups), and results in safer carbohydrate samples with higher purity. Therefore, development of medical applications for the KS oligosaccharides prepared by this method will be made easier. However, it is necessary for studies and materials applications of KSPGs to carry out both chain-elongation of the synthetic KS oligosaccharides and conjugation with $\mathrm{N}$ - and $O$-glycan linker oligosaccharides. Obviously, novel innovations are needed for conjugation of the KS oligosaccharides with linker oligosaccharides, considering the substrate specificity of KsT202. After these issues are solved, critical functions for not only KS chains but also KSPGs will be further revealed, and may open the way to the development of clinical applications for these carbohydrates.

\section{Acknowledgments}

The authors thank Seikagaku Corporation for kindly providing KsT202 used in the chemo-enzymatic synthesis of KS oligosaccharides. We further thank Prof. Hideto Watanabe (Aichi Medical University) for his help in obtaining KsT202. Finally, we would like to express our gratitude for the financial support from JSPS KAKENHI (24550134), Foundation for Promotion of Material Science and Technology of Japan (MST Foundation), and Nakanishi Scholarship Foundation to the studies using KSase II.

\section{Supplementary Material}

Supplementary material is available on TIGG publications website at https://doi.org/10.4052/tigg.1830.1E.

\section{References}

1. Hirschmann, F., Krause, F., and Papenbrock, J. (2014) Front. Plant. Sci. 5, 556.

2. Cunha, L., and Grenha, A. (2016) Mar. Drugs 14, 42.

3. Prydz, K., and Dalen, K. T. (2000) J. Cell Sci. 113, 193-205.

4. Honke, K. (2013) Proc. Jpn. Acad., Ser. B, Phys. Biol. Sci. 89, 129138.

5. For example, Stern, R., Asari, A. A., and Sugahara, K. N. (2006) Eur. J. Cell Biol. 85, 699-715.

6. For example, Qiu, H., et al. (2018) Nat. Methods 15, 889-899.

7. Miller, G. M., and Hsieh-Wilson, L. C. (2015) Exp. Neurol. 274(Pt B), 115-125.

8. Suzuki, M. (1939) J. Biochem. 30, 185-191.

9. Funderburgh, J. L. (2000) Glycobiology 10, 951-958.

10. Hart, G. W., and Lennarz, W. J. (1978) J. Biol. Chem. 253, 57955801.

11. Ziegler, C., and Mersmann, G. (1984) Biochim. Biophys. Acta 799, 203-208.

12. Seko, A., and Yamashita, K. (2004) FEBS Lett. 556, 216-220.

13. Grunwell, J. R., and Bertozzi, C. R. (2002) Biochemistry 41, 13117-
13126.

14. Akama, T. O., et al. (2000) Nat. Genet. 26, 237-241.

15. Akama, T. O., et al. (2002) J. Biol. Chem. 277, 42505-42513.

16. Yu, S.-Y., et al. (2018) J. Biol. Chem. 293, 15163-15177.

17. Zhang, H., et al. (2006) Glycobiology 16, 702-710.

18. Amado, M., et al. (1999) Biochim. Biophys. Acta 1473, 35-53.

19. Seko, A., et al. (2003) J. Biol. Chem. 278, 9150-9158.

20. Fukuta, M., et al. (1997) J. Biol. Chem. 272, 32321-32328.

21. Yusa, A., Kitajima, K., and Habuchi, O. (2006) J. Biol. Chem. 281, 20393-20403.

22. Torii, T., Fukuta, M., and Habuchi, O. (2000) Glycobiology 10, 203211.

23. Mondal, N., et al. (2018) J. Biol. Chem. 293, 7300-7314.

24. Hiraoka, N., et al. (2007) Glycobiology 17, 277-293.

25. Chandran, P. L., and Horkay, F. (2012) Acta Biomater. 8, 3-12.

26. Chen, L., et al. (2017) J. Tissue Eng. Regen. Med. 11, 602-608.

27. Kesimer, M., et al. (2013) Mucosal Immunol. 6, 379-392.

28. Geisert, E. E. Jr., et al. (1996) Int. J. Dev. Neurosci. 14, 257-267.

29. Bartholome, O., et al. (2017) Front. Mol. Neurosci. 10, 148.

30. Takeda-Uchimura, Y., et al. (2015) Exp. Neurol. 274(Pt B), 145-155.

31. Liles, M., et al. (2010) Invest. Ophthalmol. Vis. Sci. 51, 1365-1372.

32. Kadomatsu, K., and Sakamoto, K. (2014) Arch. Biochem. Biophys. 558, 36-41.

33. Foyez, T., et al. (2015) Am. J. Pathol. 185, 3053-3065.

34. Zhang, Z., et al. (2017) Proc. Natl. Acad. Sci. U.S.A. 114, E2947E2954.

35. Gonzalez-Gil, A., et al. (2018) Glycobiology 28, 786-801.

36. Mitsuoka, C., et al. (1998) J. Biol. Chem. 273, 11225-11233.

37. Stanczak, M. A., et al. (2018) J. Clin. Invest. 128, 4912-4923.

38. Nakao, H., et al. (2017) Glycobiology 34, 789-795.

39. Soares da Costa, D., Reis, R. L., and Pashkuleva, I. (2017) Annu. Rev. Biomed. Eng. 19, 1-26.

40. Kobayashi, M., et al. (1989) Tetrahedron Lett. 30, 4547-4550.

41. Kobayashi, M., et al. (1990) Carbohydr. Res. 201, 51-67.

42. Hahm, H. S., et al. (2017) Chem 2, 114-124.

43. Kitayama, K., et al. (2007) J. Biol. Chem. 282, 30085-30096.

44. Kobayashi, S., et al. (2006) Chemistry 12, 5962-5971.

45. Ohmae, M., et al. (2007) ChemBioChem 8, 1710-1720.

46. Yamazaki, Y., et al. (2016) ChemBioChem 17, 1879-1886.

47. Yamazaki, Y., Kimura, S., and Ohmae, M. (2018) Carbohydr. Res. 456, 61-68.

48. Kobayashi, S., and Ohmae, M. (2006) Adv. Polym. Sci. 194, 159210.

49. Nakazawa, K., et al. (1989) in Keratan Sulfate (Greiling, H., and Scott, J. E., eds.) pp. 99-110, The Biochemical Society, London.

50. Mark, B. L., and James, M. N. G. (2002) Can. J. Chem. 80, 10641074.

51. Igarashi, K., et al. (2014) Nat. Commun. 5, 3975.

52. Brown, G. M., et al. (1994) Biochemistry 33, 4836-4846.

53. Brown, G. M., Huckerby, T. N., and Nieduszynski, I. A. (1994) Eur. J. Biochem. 224, 281-308.

54. Davies, G. J., Wilson, K. S., and Henrissat, B. (1997) Biochem. J. 321, 557-559.

55. Yamagishi, K. S., et al. (2003) J. Biol. Chem. 278, 25766-25772.

56. Noguchi, M., Kobayashi, A., and Shoda, S. (2015) Trends Glycosci. Glycotechnol. 27, E35-E42.

57. Yamazaki, Y., et al. (2016) Carbohydr. Res. 422, 34-44. 


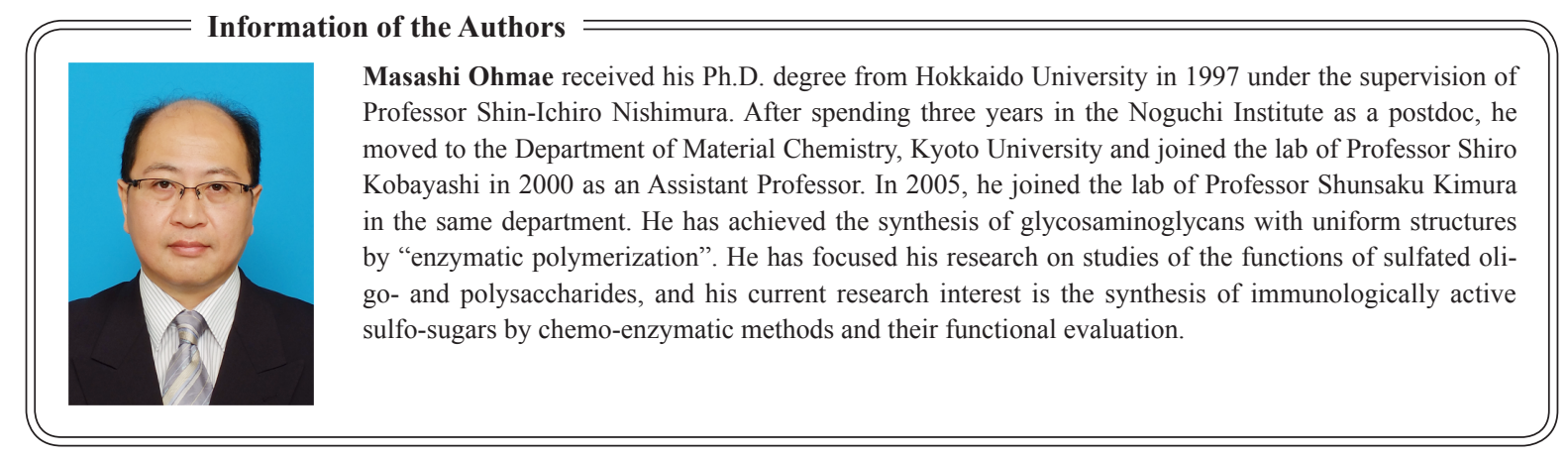

